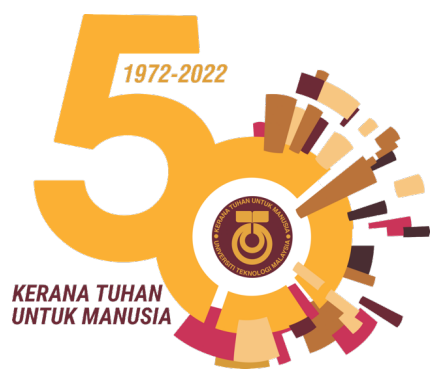

\title{
A Bibliometric Analysis of Publications of Universiti Teknologi Malaysia
}

\author{
Abdul Hakim Md Yusop ${ }^{a}$, Teong Han Chew ${ }^{b}$, Fadzlin Md Sairana, \\ Hadi Nura,c,*
}

a Center for Sustainable Nanomaterials, Ibnu Sina Institute for Scientific and Industrial Research, Universiti Teknologi Malaysia, Johor, Malaysia; b Office of Deputy Vice Chancellor (Research and Innovation), Universiti Teknologi Malaysia, Johor, Malaysia; ' Center of Advanced Materials for Renewable Energy (CAMRY), Universitas Negeri Malang, Malang, Indonesia

Abstract This study analyses the scholarly publications of Universiti Teknologi Malaysia (UTM) in conjunction with 50 years of UTM establishment. A bibliometric analysis is performed to analyse the UTM's publication and research trends over the 50 years, especially during the post-research university $(\mathrm{RU})$ period, publications impacts, subject area expertise and publications performance in comparison to other RU. Based on the analysis, engineering and computer science are the most prominent fields among the research fields in UTM in terms of a number of publications and citations thus far. Among the top 10 fields in UTM, materials science, chemical engineering, energy and chemistry areas are seen to have a high potential to further excel in citation provided that the publication numbers of these fields are further increased. The publications output for some UTM fields can still be considered low despite having a reasonable number of authors and talents. UTM is the top university in the number of publications from 2015 till 2020 in four fields, namely engineering, computer science, materials science, and chemical engineering, compared to other RU.

Keywords: bibliometric, Scopus publication, Universiti Teknologi Malaysia, research university.

\section{Introduction}

Since the proclamation of the formation of Institut Teknologi Kebangsaan (ITK) on March 14, 1972, the ITK, then declared as Universiti Teknologi Malaysia on April 1, 1975, has progressively developed and has undergone a comprehensive institutional transformation towards more impactful functional operations to remain relevant and competitive in an increasingly challenging global environment. Research is one of the criteria that UTM has vigorously prospered, as evidenced by its increasing number

*For correspondence: hadinur@utm.my

Received: 15 Jan 2022 Accepted: 20 Feb 2022

(C) Copyright Yusop et al. This article is distributed under the terms of the Creative Commons Attribution License, which permits unrestricted use and redistribution provided that the original author and source are credited. of publications every year, especially after its conferment as the research university (RU) in 2010.

Publications could be considered a medium for knowledge discussion and sharing [1] besides providing the latest updates on developing a particular research topic. Publications in Scopus- and ISI-indexed journals have always targeted Malaysia's public universities, particularly the research university (RU). Ministry of Higher Education (MOHE) has prioritized both Scopus- and ISI publications as one of the research performance indicators for Malaysia's public university [2].

Many studies have been published worldwide to analyse scholarly publications of institutions, organizations and certain particular fields using various top tiers publishers and databases such as Web of Science (WOS), Scopus, Elsevier, Science Direct, EBSCO, JSTOR, ProQuest, etc. [3-7]. Several approaches including big data analysis [8], scientometric [9] and bibliometric study [10] have been rousingly performed to thoroughly determine qualitatively and quantitatively the trend of the publications and researches of certain fields and institutions as well. Bibliometric analysis is commonly used to assess a specific field's qualitative and quantitative interest through publications analysis [1]. 
The present study uses bibliometric analysis to look in-depth at the UTM's publication and research trends over the 50 years especially the post-RU period, publications impacts, subject area expertise and publications performance compared to other RU. The publication is one of the indicators of research productivity and can be used to rank and analyse the productivity and impacts of researchers, faculties, and academic institutions [1]. Thus, there is a need to review UTM publication outputs to analyse its performance in research in conjunction with UTM's 50-years establishment as a public university in Malaysia.

\section{Methods}

Publication record was downloaded from Elsevier's Scopus, arguably the largest scientific indexed abstract and citation database. The dataset preparation stage is relatively straightforward (Figure 1) using the query functions in Scopus. The dataset was separated during the downloading stage due to the system limitation before combining everything into a total of 45,373 records. A total of 3,126 records were discarded, thus restricting the analysis to the range of 1980 (the oldest record affiliated with UTM in Scopus) to 2020.

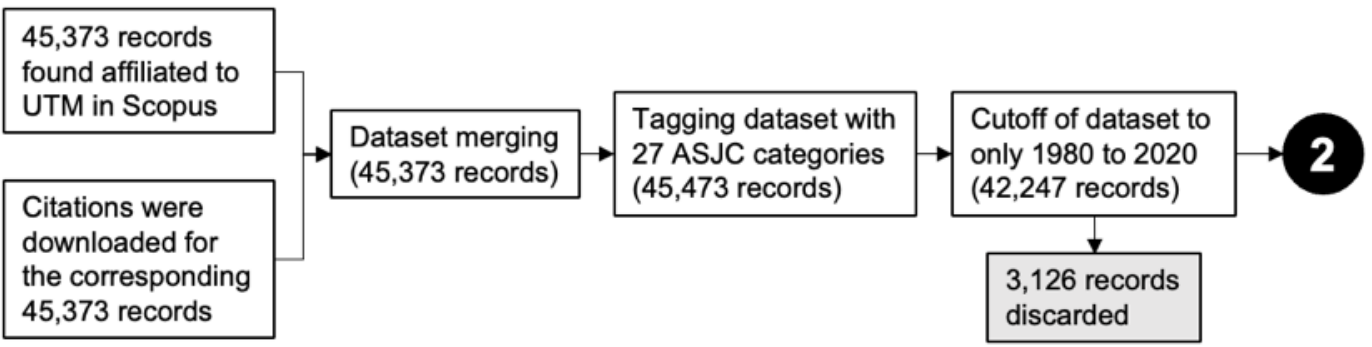

Figure 1. Dataset preparation.

Publications records in Scopus can be tagged to multiple All Science Journal Classification Codes (ASJC) which are divided into two levels of details; the subject areas and the fields. The fields can be grouped into 27 Scopus ASJC categories, as shown in Table 1. Thus, the term 'fields' hereafter used in the present study refers to the Scopus ASJC categories, and it is used interchangeably with the latter throughout this analysis article. During the analysis stage, publications tagged with multiple categories were treated as "contributing" (number of publications, number of citations, etc.) to each category accordingly. The number of authors was determined using the unique identifier (known as Author ID). Authors were grouped, and those with low publication count and not affiliated to UTM were removed (Figure 2).

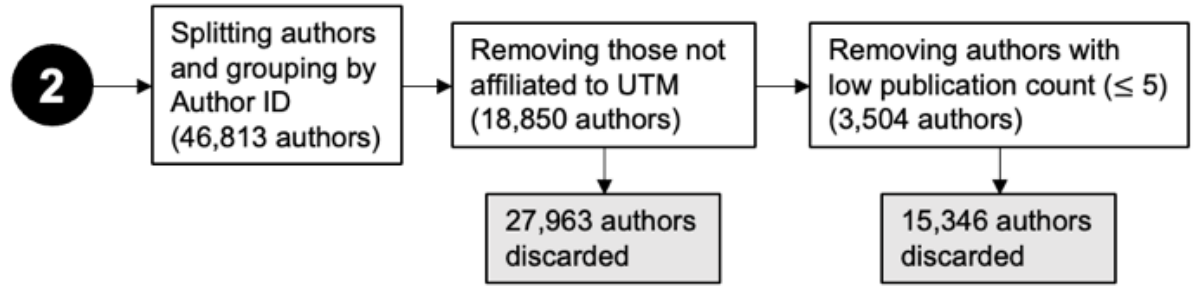

Figure 2. Author(s) identification and screening.

The second dataset used in the analysis comes from UTM's internal database, our integrated research grant management system (known as Research and Development Information System, RADIS, https://radis.utm.my/). RADIS was fully launched in 2013 (the then version 3.0) with continuous updates over the years before being completely refreshed in 2020 as version 4.0. Research grants secured by any researchers in UTM were registered in RADIS and one of the categorizations used is Field of Research (FOR) and Socio-Economic Objective (SEO). 
Table 1. Scopus ASJC categories.

\begin{tabular}{ll}
\hline Description & Code \\
\hline Engineering & ENGI \\
Social Sciences & SOCI \\
Computer Science & COMP \\
Agricultural and Biological Sciences & AGRI \\
Biochemistry, Genetics and Molecular Biology & BIOC \\
Environmental Science & ENVI \\
Medicine & MEDI \\
Earth and Planetary Sciences & EART \\
Physics and Astronomy & PHYS \\
Economics, Econometrics and Finance & ECON \\
Chemistry & CHEM \\
Arts and Humanities & ARTS \\
Materials Science & MATE \\
Business, Management and Accounting & BUSI \\
Mathematics & MATH \\
Energy & ENER \\
Decision Sciences & DECI \\
Immunology and Microbiology & IMMU \\
Chemical Engineering & CENG \\
Psychology & PSYC \\
Dentistry & DENT \\
Neuroscience & NEUR \\
Nursing & NURS \\
Health Professions & HEAL \\
Veterinary & VETE \\
Pharmacology, Toxicology and Pharmaceutics & PHAR \\
Multidisciplinary & MULT \\
\hline &
\end{tabular}

This classification is based on the Malaysian Research and Development System (MRDS) [11] (only the $6^{\text {th }}$ Edition was implemented in RADIS). A mapping was created between Scopus's ASJC categories and MRDS's FOR, in order for the analysis to be cross-examined between publications records and grants records. Granted, the mapping is not perfect by any means due to the different approaches and categorization used by Scopus and MRDS. MRDS's FOR categorization system employed four levels of detail (Figure 3), and a combination of FOR Group and FOR Area was used to map the research grant to Scopus's ASJC categories.

$\begin{array}{lll}\text { EXAMPLE 'FOR' } & : \text { F1020104 } \\ \text { SYSTEM OF CLASSIFICATION }: \text { F } & \\ \text { DIVISION (1 Digit ) } & : 1 & : \text { Natural Sciences } \\ \text { CATEGORY (2 Digit) } & : 02 & : \text { Physical Sciences } \\ \text { GROUP (2 Digit) } & : 01 & : \text { Astronomy and Astrophysics } \\ \text { AREA (2 Digit) } & : 04 & : \text { Stellar System }\end{array}$

Figure 3. Example of FOR categorization in MRDC $6^{\text {th }}$ Edition

Unlike the publications which used one (publication) to many (ASJC categories) tagging, the research grant only uses one-to-one tagging. Due to the sensitivity of the information, raw values (i.e., funding amount) were not disclosed. However, the values used in the analysis underwent a min-max normalization, resulting in a standard scale of 0 to 1 , especially during the analysis in the spider charts (of each ASJC category). 
The amount of funding of each research grant was not used in totality but was divided into two groups; the amount allocated for salary pay-out or emolument (denoted as talent) and the amount allocated for equipment purchase (denoted as equipment). The fact that the Government of Malaysia, via the Ministry of Finance, exercise a standard practice of financial coding to differentiate the different source of income and expenditure makes the segregation of talent and equipment more accessible. Assumptions were made that the amount allocated for talent would directly influence supporting staff (research officers, graduate students, etc.) in carrying out the research activities. That equipment would reflect the amount of money spent on the hardware and software directly used in the research.

\section{Results}

\section{Publications trend by fields}

Table 2 and Table 3 show the number of Scopus-indexed publications of Universiti Teknologi Malaysia (UTM) from the year 1980 till the year 2020 based on different fields (denoted as ASJC categories in Table 1) in the descending order from left to right. It can be seen that there has been a remarkable increase in publications of the majority of the fields from 2010 onwards. The award of research-university status (RU) for UTM in 2010 might lead to this notable increase as the top six of the fields that constantly soar upwards are Engineering (ENGI), Computer Science (COMP), Material Sciences (MATE), Physics and Astronomy (PHYS), Chemical Engineering (CENG) and Environmental Science (ENVI).

In the present study, the number of UTM's Scopus-indexed publications based on the different fields from the year 1980 to 2020 is categorized into four classes, namely primary $(>10,000$ publications), high potential (between 4,000 and 10,000 publications), potential (between 1,000 and 4,000 publications) and emerging (<1,000 publications). As indicated in Table 4, there are two primary fields with the highest Scopus publications up to 2020; Engineering (ENGI) with 20,043 publications, followed by Computer Science (COMP) with 11,142 publications. Materials Sciences (MATE), Physics and Astronomy (PHYS), Environmental Sciences (ENVI), Chemical Engineering (CENG) and Mathematics (MATH) are the fields constituting the high-potential secondary cohort. Energy (ENER), Chemistry (CHEM) and Social Sciences $(\mathrm{SOCl})$ are the top three for the potential tertiary group. The fourth group, namely the emerging cohort, is led by Decision Sciences (DECl), Economics, Econometrics and Finance (ECON) and Arts and Humanities (ARTS). Nursing (NURS), Dentistry (DENT) and Veterinary (VETE) possess the least number of publications, and this is highly reasonable as these fields have not been a prominent field of UTM thus far. The progression rate of the publication of the areas in UTM from 2010 to 2020 is measured using the compound annual growth rate (CAGR) formula and is summarized in Table 4.

\section{Citation analysis}

Table 5 and Table 6 show the number of citations from Scopus-indexed publications of UTM up to the year 2020. Again, Engineering (ENGI) tops the group, followed by Material Sciences (MATE), Computer Sciences (COMP), Chemical Engineering (CENG) and Chemistry (CHEM). Apparently, Physics and Astronomy (PHYS), which ranked $4^{\text {th }}$ in a total number of publications, descends to 8th place in terms of the total number of citations generated. Intriguingly, Chemical Engineering (CENG) and Chemistry (CHEM) have a better rank in the total citation ( $4^{\text {th }}$ and $5^{\text {th }}$, respectively) despite being ranked $6^{\text {th }}$ and 9 th in total publications, respectively. This finding connotes that a high publication does not necessarily lead to a high citation as the citation highly depends on the quality of the published articles, in general.

The impact of the publication is then assessed as the ratio of total citations to the number of publications for each ASJC category. Table 7 displays the ratio of total citations to the numbers of publications for the top ten ASJC categories selected from 2010 (the conferment of UTM as the RU) up to the year 2020. In general, the ratios of citations to publications show a decreasing trend from 2010 to 2020 for all fields as the ratio remarkably starts to descend in 2016 or 2017. Some ASJC categories such as Energy (ENEG), Chemistry (CHEM), Environmental Sciences (ENVI), Chemical Engineering (CENG) and Material Sciences (MATE) propelled higher in citation/publication ratio particularly in the year 2010 to 2015 period and in fact, surpassing those of Engineering (ENGI) and Computer Sciences (COMP). While a higher number of publications could result in a higher probability of being cited, Table 7 shows a good example that citations can be very field-dependent. This is seen for Engineering (ENGI) and Computer Sciences (COMP), which possess a moderate citation per publication ratio, despite being two of the highest ASJC categories in publication volume. 
Table 2. Number of publications by year by ASJC Categories (Part 1) *Colour represents the "intensity" based on the numbers of publication with blue as cold and red as hot.

\begin{tabular}{|c|c|c|c|c|c|c|c|c|c|c|c|c|c|}
\hline Year & ENGI & COMP & MATE & PHYS & ENVI & CENG & MATH & ENER & CHEM & SOCl & BIOC & EART & BUSI \\
\hline 1980 & 0 & 0 & 0 & 0 & 0 & 0 & 0 & 0 & 0 & 0 & 0 & 1 & 0 \\
\hline 1982 & 0 & 0 & 0 & 0 & 0 & 0 & 0 & 0 & 0 & 1 & 0 & 0 & 0 \\
\hline 1984 & 3 & 0 & 0 & 0 & 0 & 0 & 1 & 0 & 0 & 0 & 0 & 0 & 0 \\
\hline 1985 & 3 & 0 & 1 & 1 & 1 & 0 & 0 & 0 & 0 & 1 & 0 & 0 & 0 \\
\hline 1986 & 4 & 1 & 0 & 0 & 0 & 0 & 0 & 0 & 0 & 0 & 0 & 0 & 0 \\
\hline 1987 & 5 & 0 & 0 & 0 & 2 & 0 & 0 & 0 & 0 & 0 & 0 & 0 & 0 \\
\hline 1988 & 6 & 0 & 3 & 1 & 1 & 0 & 0 & 0 & 0 & 2 & 0 & 1 & 0 \\
\hline 1989 & 4 & 0 & 1 & 1 & 1 & 0 & 0 & 0 & 2 & 1 & 0 & 1 & 0 \\
\hline 1990 & 8 & 0 & 1 & 0 & 0 & 1 & 0 & 1 & 0 & 0 & 0 & 0 & 0 \\
\hline 1991 & 5 & 2 & 1 & 2 & 1 & $\overline{0}$ & 2 & 0 & 0 & 1 & 0 & 3 & 0 \\
\hline 1992 & 6 & 4 & 2 & 3 & 0 & 0 & 2 & 0 & 0 & 2 & 0 & 0 & 1 \\
\hline 1993 & 9 & 2 & 0 & 1 & 1 & 1 & 1 & 1 & 2 & 2 & 0 & 0 & 0 \\
\hline 1994 & 16 & 5 & 7 & 4 & 3 & 5 & 3 & 5 & 6 & 1 & 3 & 0 & 0 \\
\hline 1995 & 17 & 6 & 3 & 4 & 0 & 2 & 3 & 1 & 5 & 0 & 1 & 0 & 1 \\
\hline 1996 & 13 & 8 & 5 & 5 & 6 & 2 & 4 & 3 & 7 & 1 & 4 & 2 & 1 \\
\hline 1997 & 25 & 9 & 11 & 9 & 4 & 5 & 8 & 4 & 14 & 2 & 3 & 4 & 2 \\
\hline 1998 & 26 & 14 & 8 & 8 & 4 & 3 & 10 & 6 & 10 & 1 & 3 & 0 & 0 \\
\hline 1999 & 23 & 8 & 8 & 3 & 2 & 2 & 4 & 5 & 11 & 0 & 2 & 2 & 0 \\
\hline 2000 & 82 & 63 & 25 & 14 & 2 & 5 & 8 & 7 & 14 & 4 & 0 & 2 & 3 \\
\hline 2001 & 23 & 28 & 9 & 4 & 4 & 2 & 3 & 6 & 6 & 3 & 1 & 5 & 3 \\
\hline 2002 & 73 & 33 & 28 & 9 & 13 & 8 & 14 & 9 & 10 & 17 & 6 & 1 & 0 \\
\hline 2003 & 78 & 63 & 15 & 10 & 7 & 14 & 12 & 18 & 15 & 15 & 3 & 3 & 6 \\
\hline 2004 & 115 & 41 & 19 & 16 & 17 & 21 & 16 & 9 & 22 & 5 & 9 & 5 & 2 \\
\hline 2005 & 164 & 67 & 23 & 20 & 21 & 25 & 18 & 11 & 24 & 6 & 8 & 6 & 5 \\
\hline 2006 & 140 & 77 & 45 & 27 & 18 & 45 & 16 & 28 & 42 & 16 & 15 & 14 & 5 \\
\hline 2007 & 190 & 117 & 47 & 36 & 36 & 38 & 33 & 27 & 32 & 44 & 16 & 5 & 6 \\
\hline 2008 & 376 & 346 & 108 & 82 & 23 & 41 & 83 & 75 & 35 & 51 & 28 & 11 & 14 \\
\hline 2009 & 346 & 470 & 145 & 118 & 58 & 48 & 104 & 30 & 60 & 60 & 50 & 41 & 31 \\
\hline 2010 & 570 & 457 & 219 & 213 & 77 & 101 & 150 & 81 & 85 & 106 & 86 & 48 & 53 \\
\hline 2011 & 840 & 628 & 250 & 289 & 100 & 148 & 285 & 108 & 122 & 78 & 110 & 62 & 92 \\
\hline 2012 & 1128 & 687 & 356 & 370 & 164 & 235 & 297 & 192 & 216 & 125 & 103 & 90 & 82 \\
\hline 2013 & 1693 & 762 & 471 & 362 & 258 & 283 & 361 & 287 & 230 & 234 & 234 & 145 & 67 \\
\hline 2014 & 2321 & 921 & 491 & 454 & 423 & 311 & 315 & 358 & 325 & 229 & 223 & 235 & 151 \\
\hline 2015 & 2265 & 921 & 540 & 489 & 420 & 399 & 345 & 369 & 390 & 384 & 185 & 158 & 226 \\
\hline 2016 & 1803 & 826 & 567 & 588 & 405 & 295 & 276 & 302 & 368 & 350 & 143 & 139 & 226 \\
\hline 2017 & 1937 & 1197 & 593 & 600 & 490 & 513 & 533 & 530 & 325 & 557 & 141 & 102 & 186 \\
\hline 2018 & 1807 & 1161 & 701 & 592 & 597 & 599 & 261 & 311 & 353 & 278 & 348 & 245 & 181 \\
\hline 2019 & 2025 & 1279 & 913 & 711 & 601 & 473 & 384 & 366 & 325 & 400 & 146 & 291 & 321 \\
\hline 2020 & 1882 & 939 & 1077 & 704 & 616 & 521 & 429 & 388 & 411 & 308 & 329 & 282 & 199 \\
\hline
\end{tabular}


Table 3. Number of publications by year by ASJC Categories (Part 2) *Colour represents the "intensity" based on the numbers of publication with blue as cold and red as hot.

\begin{tabular}{|c|c|c|c|c|c|c|c|c|c|c|c|c|c|c|}
\hline Year & AGRI & MULT & MEDI & DECI & ECON & ARTS & PHAR & IMMU & PSYC & HEAL & NEUR & NURS & VETE & DENT \\
\hline 1980 & 0 & 0 & 0 & 0 & 0 & 0 & 0 & 0 & 0 & 0 & 0 & 0 & 0 & 0 \\
\hline 1982 & 0 & 0 & 0 & 0 & 0 & 0 & 0 & 0 & 0 & 0 & 0 & 0 & 0 & 0 \\
\hline 1984 & 0 & 0 & 0 & 0 & 0 & 0 & 0 & 0 & 0 & 0 & 0 & 0 & 0 & 0 \\
\hline 1985 & 0 & 0 & 0 & 0 & 0 & 0 & 0 & 0 & 0 & 0 & 0 & 0 & 0 & 0 \\
\hline 1986 & 0 & 0 & 0 & 0 & 0 & 0 & 0 & 0 & 0 & 0 & 0 & 0 & 0 & 0 \\
\hline 1987 & 0 & 0 & 0 & 0 & 0 & 0 & 0 & 0 & 0 & 0 & 0 & 0 & 0 & 0 \\
\hline 1988 & 0 & 0 & 0 & 0 & 0 & 0 & 0 & 0 & 0 & 0 & 0 & 0 & 0 & 0 \\
\hline 1989 & 0 & 0 & 0 & 0 & 0 & 0 & 0 & 0 & 0 & 0 & 0 & 0 & 0 & 0 \\
\hline 1990 & 0 & 0 & 0 & 0 & 0 & 0 & 0 & 0 & 0 & 0 & 0 & 0 & 0 & 0 \\
\hline 1991 & 0 & 0 & 0 & 0 & 0 & 0 & 0 & 0 & 0 & 0 & 0 & 0 & 0 & 0 \\
\hline 1992 & 0 & 0 & 0 & 0 & 0 & 0 & 0 & 0 & 0 & 0 & 0 & 0 & 0 & 0 \\
\hline 1993 & 0 & 0 & 1 & 0 & 1 & 0 & 0 & 0 & 0 & 0 & 0 & 0 & 0 & 0 \\
\hline 1994 & 1 & 0 & 3 & 0 & 0 & 0 & 1 & 0 & 0 & 0 & 0 & 0 & 0 & 0 \\
\hline 1995 & 1 & 0 & 0 & 0 & 0 & 0 & 0 & 0 & 0 & 0 & 0 & 0 & 0 & 0 \\
\hline 1996 & 0 & 0 & 1 & 0 & 0 & 0 & 1 & 0 & 0 & 0 & 0 & 0 & 0 & 0 \\
\hline 1997 & 0 & 0 & 2 & 1 & 0 & 0 & 1 & 0 & 0 & 0 & 0 & 0 & 0 & 0 \\
\hline 1998 & 0 & 0 & 2 & 0 & 0 & 1 & 1 & 0 & 0 & 0 & 0 & 0 & 0 & 0 \\
\hline 1999 & 0 & 0 & 1 & 1 & 0 & 0 & 0 & 1 & 0 & 0 & 0 & 0 & 0 & 0 \\
\hline 2000 & 0 & 0 & 0 & 2 & 0 & 0 & 0 & 0 & 0 & 0 & 0 & 0 & 0 & 0 \\
\hline 2001 & 0 & 1 & 2 & 2 & 0 & 0 & 0 & 0 & 0 & 0 & 0 & 0 & 0 & 0 \\
\hline 2002 & 0 & 0 & 2 & 0 & 0 & 1 & 1 & 0 & 0 & 0 & 0 & 0 & 0 & 0 \\
\hline 2003 & 5 & 3 & 1 & 2 & 1 & 0 & 0 & 0 & 0 & 0 & 0 & 0 & 0 & 0 \\
\hline 2004 & 3 & 0 & 5 & 3 & 0 & 1 & 1 & 3 & 0 & 0 & 0 & 0 & 0 & 0 \\
\hline 2005 & 5 & 2 & 7 & 6 & 0 & 5 & 2 & 2 & 0 & 2 & 0 & 0 & 0 & 0 \\
\hline 2006 & 5 & 9 & 5 & 3 & 1 & 2 & 2 & 4 & 0 & 0 & 0 & 1 & 0 & 0 \\
\hline 2007 & 3 & 15 & 8 & 22 & 2 & 1 & 4 & 2 & 1 & 0 & 4 & 0 & 0 & 0 \\
\hline 2008 & 9 & 17 & 11 & 2 & 2 & 4 & 3 & 5 & 0 & 1 & 1 & 1 & 0 & 0 \\
\hline 2009 & 39 & 29 & 20 & 27 & 7 & 1 & 9 & 6 & 0 & 0 & 2 & 1 & 0 & 0 \\
\hline 2010 & 38 & 65 & 20 & 31 & 9 & 5 & 16 & 2 & 10 & 2 & 1 & 0 & 0 & 1 \\
\hline 2011 & 90 & 116 & 32 & 36 & 13 & 12 & 21 & 27 & 10 & 3 & 8 & 0 & 1 & 0 \\
\hline 2012 & 58 & 97 & 30 & 26 & 19 & 26 & 26 & 29 & 5 & 2 & 1 & 3 & 1 & 2 \\
\hline 2013 & 100 & 161 & 75 & 37 & 40 & 22 & 36 & 31 & 4 & 8 & 2 & 0 & 1 & 1 \\
\hline 2014 & 112 & 123 & 63 & 34 & 57 & 64 & 45 & 41 & 9 & 9 & 8 & 0 & 0 & 3 \\
\hline 2015 & 120 & 71 & 133 & 124 & 158 & 113 & 66 & 54 & 9 & 11 & 16 & 4 & 0 & 0 \\
\hline 2016 & 132 & 101 & 73 & 80 & 60 & 92 & 59 & 33 & 14 & 14 & 15 & 3 & 1 & 0 \\
\hline 2017 & 150 & 53 & 81 & 69 & 59 & 156 & 27 & 19 & 13 & 11 & 8 & 1 & 0 & 1 \\
\hline 2018 & 120 & 53 & 132 & 113 & 81 & 30 & 32 & 25 & 12 & 7 & 5 & 1 & 0 & 0 \\
\hline 2019 & 130 & 69 & 215 & 106 & 49 & 45 & 44 & 29 & 9 & 11 & 6 & 2 & 2 & 2 \\
\hline 2020 & 202 & 122 & 114 & 104 & 70 & 30 & 127 & 39 & 17 & 11 & 5 & 11 & 4 & 0 \\
\hline
\end{tabular}


Table 4. Number of publications, publication growth rate and number of researchers for each field (*Total publication from 1980 to 2020 , CAGR is compound annual growth rate, number of authors derived from the corresponding set of publications based on unique Author ID).

\begin{tabular}{|c|c|c|c|}
\hline ASJC Category & $\begin{array}{c}\text { Total } \\
\text { Publication }\end{array}$ & $\begin{array}{c}\text { CAGR (\%) 2010- } \\
2020\end{array}$ & No. of Authors \\
\hline \multicolumn{4}{|l|}{ Primary } \\
\hline Engineering (ENGI) & 20,031 & 14 & 3,299 \\
\hline Computer sciences (COMP) & 11,142 & 8 & 2,360 \\
\hline \multicolumn{4}{|l|}{ High Potential } \\
\hline Material sciences (MATE) & 6,693 & 19 & 2,171 \\
\hline Physics and Astronomy (PHYS) & 5,750 & 14 & 2,063 \\
\hline Environmental Science (ENVI) & 4,376 & 26 & 1,964 \\
\hline Chemical Engineering (CENG) & 4,146 & 20 & 1,693 \\
\hline Mathematics (MATH) & 3,981 & 12 & 1,812 \\
\hline \multicolumn{4}{|l|}{ Potential } \\
\hline Energy (ENER) & 3,538 & 19 & 1,668 \\
\hline Chemistry (CHEM) & 3,467 & 19 & 1,442 \\
\hline Social sciences $(\mathrm{SOCl})$ & 3,258 & 13 & 1,302 \\
\hline Biochemistry, Genetics and Molecular Biology (BIOC) & 2,200 & 16 & 1,375 \\
\hline Earth and Planetary Sciences (EART) & 1,904 & 22 & 884 \\
\hline Business, Management and Accounting (BUSI) & 1,864 & 16 & 915 \\
\hline Agricultural and Biological Sciences (AGRI) & 1,323 & 20 & 914 \\
\hline Multidisciplinary (MULT) & 1,107 & 7 & 917 \\
\hline Medicine (MEDI) & 1,039 & 21 & 788 \\
\hline \multicolumn{4}{|l|}{ Emerging } \\
\hline Decision Sciences (DECl) & 831 & 14 & 574 \\
\hline Economics, Econometrics and Finance (ECON) & 629 & 26 & 384 \\
\hline Arts and Humanities (ARTS) & 611 & 22 & 355 \\
\hline Pharmacology, Toxicology and Pharmaceutics (PHAR) & 525 & 26 & 360 \\
\hline Immunology and Microbiology (IMMU) & 352 & 39 & 262 \\
\hline Psychology (PSYC) & 113 & 6 & 96 \\
\hline Health Professions (HEAL) & 92 & 21 & 102 \\
\hline Neuroscience (NEUR) & 82 & 20 & 94 \\
\hline Nursing (NURS) & 28 & $\mathrm{~N} / \mathrm{A}$ & 36 \\
\hline Dentistry (DENT) & 10 & -100 & 11 \\
\hline Veterinary (VETE) & 10 & $\mathrm{~N} / \mathrm{A}$ & 10 \\
\hline
\end{tabular}


Table 5. Citations generated by publications by year by ASJC categories (Part 1) *Colour represents the "intensity" based on the numbers of publication with blue as cold and red as hot. (*Year refers to the year of citation received)

\begin{tabular}{|c|c|c|c|c|c|c|c|c|c|c|c|}
\hline Year & ENGI & MATE & COMP & CENG & CHEM & ENER & ENVI & PHYS & BIOC & MATH & BUSI \\
\hline$<1990$ & 12 & 12 & 0 & 0 & 0 & 0 & 0 & 16 & 0 & 1 & 0 \\
\hline 1990 & 0 & 0 & 0 & 0 & 0 & 0 & 0 & 10 & 0 & 0 & 0 \\
\hline 1991 & 1 & 1 & 0 & 0 & 2 & 0 & 2 & 7 & 0 & 0 & 0 \\
\hline 1992 & 0 & 1 & 0 & 0 & 3 & 0 & 1 & 9 & 0 & 1 & 0 \\
\hline 1993 & 0 & 0 & 0 & 0 & 2 & 0 & 1 & 3 & 0 & 0 & 0 \\
\hline 1994 & 5 & 7 & 0 & 0 & 5 & 1 & 2 & 8 & 1 & 3 & 0 \\
\hline 1995 & 2 & 4 & 0 & 6 & 8 & 8 & 2 & 8 & 0 & 0 & 0 \\
\hline 1996 & 6 & 5 & 1 & 11 & 4 & 9 & 0 & 14 & 1 & 7 & 0 \\
\hline 1997 & 14 & 11 & 1 & 15 & 32 & 13 & 13 & 12 & 12 & 7 & 1 \\
\hline 1998 & 25 & 8 & 4 & 21 & 29 & 16 & 7 & 15 & 14 & 9 & 0 \\
\hline 1999 & 13 & 13 & 5 & 18 & 54 & 14 & 9 & 14 & 12 & 7 & 1 \\
\hline 2000 & 21 & 43 & 7 & 17 & 95 & 11 & 8 & 11 & 17 & 9 & 1 \\
\hline 2001 & 28 & 43 & 17 & 25 & 74 & 15 & 13 & 12 & 17 & 9 & 3 \\
\hline 2002 & 32 & 84 & 15 & 41 & 118 & 16 & 12 & 20 & 18 & 12 & 7 \\
\hline 2003 & 59 & 108 & 24 & 46 & 116 & 17 & 12 & 29 & 25 & 15 & 7 \\
\hline 2004 & 81 & 157 & 40 & 82 & 171 & 28 & 31 & 39 & 59 & 20 & 7 \\
\hline 2005 & 123 & 209 & 59 & 106 & 248 & 36 & 35 & 60 & 63 & 45 & 30 \\
\hline 2006 & 274 & 275 & 123 & 229 & 329 & 77 & 85 & 122 & 118 & 54 & 65 \\
\hline 2007 & 353 & 327 & 156 & 243 & 333 & 68 & 114 & 130 & 136 & 73 & 94 \\
\hline 2008 & 494 & 473 & 233 & 378 & 482 & 101 & 163 & 218 & 201 & 102 & 117 \\
\hline 2009 & 819 & 663 & 393 & 522 & 628 & 148 & 304 & 339 & 314 & 157 & 176 \\
\hline 2010 & 1681 & 1105 & 1001 & 765 & 862 & 273 & 409 & 614 & 452 & 346 & 183 \\
\hline 2011 & 2549 & 1502 & 1591 & 1129 & 1286 & 462 & 672 & 860 & 606 & 555 & 235 \\
\hline 2012 & 3924 & 2313 & 2240 & 1638 & 1716 & 822 & 1034 & 1476 & 835 & 790 & 298 \\
\hline 2013 & 5441 & 3099 & 2906 & 2444 & 2569 & 1508 & 1498 & 2054 & 1124 & 1093 & 413 \\
\hline 2014 & 8132 & 4435 & 3789 & 3536 & 3779 & 2867 & 2627 & 2946 & 1627 & 1576 & 627 \\
\hline 2015 & 11045 & 5878 & 4740 & 4759 & 5133 & 4065 & 3870 & 4044 & 2065 & 1825 & 865 \\
\hline 2016 & 14338 & 7482 & 5708 & 6402 & 6627 & 5765 & 5679 & 4699 & 2603 & 2180 & 1359 \\
\hline 2017 & 18103 & 9208 & 7319 & 7892 & 8096 & 8051 & 7172 & 5899 & 3063 & 2628 & 2076 \\
\hline 2018 & 23084 & 11343 & 9916 & 9903 & 9646 & 10054 & 9471 & 7271 & 3940 & 3129 & 2874 \\
\hline 2019 & 30163 & 14673 & 13822 & 12403 & 11484 & 13227 & 12497 & 9615 & 4894 & 4173 & 3847 \\
\hline 2020 & 36382 & 18399 & 16396 & 15319 & 13668 & 15818 & 16189 & 11812 & 5610 & 5200 & 5281 \\
\hline
\end{tabular}


Table 6. Citations generated by publications by year by ASJC categories (Part 2) *Colour represents the "intensity" based on the numbers of publication with blue as cold and red as hot.

\begin{tabular}{|c|c|c|c|c|c|c|c|c|c|c|c|c|c|c|c|c|}
\hline Year & EART & SOCI & AGRI & MULT & MEDI & DECI & IMMU & PHAR & ECON & ARTS & PSYC & HEAL & NEUR & NURS & DENT & VETE \\
\hline$<1990$ & 0 & 2 & 0 & 0 & 0 & 0 & 0 & 0 & 0 & 0 & 0 & 0 & 0 & 0 & 0 & 0 \\
\hline 1990 & 0 & 1 & 0 & 0 & 0 & 0 & 0 & 0 & 0 & 0 & 0 & 0 & 0 & 0 & 0 & 0 \\
\hline 1991 & 0 & 2 & 0 & 0 & 0 & 0 & 0 & 0 & 0 & 0 & 0 & 0 & 0 & 0 & 0 & 0 \\
\hline 1992 & 0 & 3 & 0 & 0 & 0 & 0 & 0 & 0 & 0 & 0 & 0 & 0 & 0 & 0 & 0 & 0 \\
\hline 1993 & 0 & 4 & 0 & 0 & 0 & 0 & 0 & 0 & 0 & 0 & 0 & 0 & 0 & 0 & 0 & 0 \\
\hline 1994 & 0 & 1 & 0 & 0 & 2 & 0 & 0 & 1 & 0 & 0 & 0 & 0 & 0 & 0 & 0 & 0 \\
\hline 1995 & 0 & 1 & 0 & 0 & 2 & 0 & 0 & 0 & 0 & 0 & 0 & 0 & 0 & 0 & 0 & 0 \\
\hline 1996 & 0 & 5 & 1 & 0 & 0 & 0 & 0 & 0 & 2 & 0 & 0 & 0 & 0 & 0 & 0 & 0 \\
\hline 1997 & 0 & 3 & 3 & 0 & 7 & 0 & 0 & 1 & 3 & 0 & 0 & 0 & 0 & 0 & 0 & 0 \\
\hline 1998 & 1 & 5 & 3 & 0 & 6 & 0 & 0 & 1 & 2 & 1 & 0 & 0 & 0 & 0 & 0 & 0 \\
\hline 1999 & 0 & 1 & 1 & 0 & 7 & 1 & 0 & 2 & 0 & 1 & 0 & 0 & 0 & 0 & 0 & 0 \\
\hline 2000 & 1 & 5 & 3 & 0 & 4 & 0 & 0 & 3 & 1 & 0 & 0 & 0 & 0 & 0 & 0 & 0 \\
\hline 2001 & 2 & 3 & 3 & 0 & 4 & 2 & 1 & 0 & 0 & 3 & 0 & 0 & 0 & 0 & 0 & 0 \\
\hline 2002 & 1 & 3 & 2 & 0 & 6 & 2 & 1 & 2 & 0 & 3 & 0 & 0 & 0 & 0 & 0 & 0 \\
\hline 2003 & 0 & 3 & 2 & 0 & 6 & 2 & 1 & 1 & 1 & 0 & 0 & 0 & 0 & 0 & 0 & 0 \\
\hline 2004 & 0 & 6 & 3 & 2 & 13 & 11 & 0 & 4 & 0 & 5 & 0 & 0 & 0 & 0 & 0 & 0 \\
\hline 2005 & 9 & 8 & 5 & 6 & 9 & 21 & 2 & 5 & 0 & 5 & 0 & 1 & 0 & 0 & 0 & 0 \\
\hline 2006 & 11 & 5 & 10 & 31 & 14 & 31 & 8 & 12 & 0 & 4 & 0 & 1 & 0 & 0 & 0 & 0 \\
\hline 2007 & 13 & 8 & 8 & 49 & 19 & 31 & 27 & 14 & 0 & 5 & 0 & 2 & 0 & 2 & 0 & 0 \\
\hline 2008 & 28 & 10 & 20 & 37 & 24 & 38 & 41 & 13 & 2 & 5 & 2 & 7 & 5 & 3 & 0 & 0 \\
\hline 2009 & 34 & 34 & 29 & 36 & 61 & 69 & 40 & 28 & 7 & 9 & 3 & 7 & 7 & 1 & 0 & 0 \\
\hline 2010 & 88 & 100 & 68 & 83 & 110 & 105 & 58 & 41 & 12 & 10 & 4 & 14 & 11 & 1 & 0 & 0 \\
\hline 2011 & 176 & 156 & 147 & 177 & 135 & 104 & 75 & 72 & 17 & 17 & 10 & 14 & 8 & 1 & 0 & 2 \\
\hline 2012 & 306 & 272 & 285 & 228 & 207 & 165 & 153 & 127 & 33 & 19 & 21 & 15 & 11 & 6 & 1 & 3 \\
\hline 2013 & 385 & 375 & 386 & 383 & 270 & 172 & 178 & 160 & 74 & 58 & 38 & 34 & 11 & 4 & 12 & 10 \\
\hline 2014 & 707 & 674 & 576 & 461 & 330 & 263 & 236 & 225 & 118 & 157 & 51 & 42 & 7 & 13 & 17 & 3 \\
\hline 2015 & 1107 & 883 & 808 & 564 & 469 & 406 & 372 & 269 & 181 & 180 & 63 & 78 & 33 & 16 & 14 & 5 \\
\hline 2016 & 1522 & 1185 & 1142 & 711 & 665 & 519 & 578 & 384 & 280 & 237 & 103 & 88 & 69 & 25 & 17 & 8 \\
\hline 2017 & 1794 & 1637 & 1539 & 958 & 834 & 687 & 724 & 535 & 415 & 360 & 158 & 142 & 115 & 27 & 20 & 5 \\
\hline 2018 & 2473 & 2670 & 1921 & 1114 & 1155 & 868 & 824 & 615 & 626 & 594 & 219 & 194 & 170 & 40 & 22 & 5 \\
\hline 2019 & 3919 & 3731 & 2609 & 1380 & 1447 & 1177 & 877 & 812 & 849 & 654 & 300 & 242 & 191 & 46 & 17 & 7 \\
\hline 2020 & 5323 & 4874 & 3477 & 1735 & 1988 & 1340 & 962 & 1148 & 1215 & 704 & 387 & 259 & 192 & 53 & 20 & 13 \\
\hline
\end{tabular}


Table 7. Citations per publications for the UTM's top 10 ASJC categories (based on total publications).

\begin{tabular}{|c|c|c|c|c|c|c|c|c|c|c|}
\hline Year & ENGI & COMP & MATE & PHYS & ENVI & CENG & MATH & ENER & CHEM & SOCI \\
\hline 2010 & 11.8 & 8.9 & 18.3 & 9.5 & 26.2 & 31.4 & 9.2 & 20.6 & 38.5 & 6.3 \\
\hline 2011 & 10.8 & 8.7 & 20.5 & 10.7 & 34.5 & 31.6 & 8.1 & 42.0 & 33.8 & 9.0 \\
\hline 2012 & 13.1 & 8.1 & 19.9 & 14.6 & 31.6 & 27.9 & 9.7 & 33.6 & 33.1 & 7.4 \\
\hline 2013 & 10.6 & 7.5 & 18.1 & 15.8 & 25.5 & 24.4 & 6.0 & 29.9 & 29.9 & 6.1 \\
\hline 2014 & 8.9 & 7.4 & 19.1 & 15.6 & 19.1 & 25.5 & 7.5 & 25.2 & 26.0 & 7.9 \\
\hline 2015 & 8.9 & 8.7 & 18.7 & 11.7 & 23.1 & 24.3 & 6.6 & 24.4 & 23.7 & 5.7 \\
\hline 2016 & 8.3 & 7.5 & 13.4 & 8.7 & 17.2 & 16.9 & 7.9 & 25.4 & 16.5 & 5.4 \\
\hline 2017 & 6.6 & 5.1 & 9.0 & 7.4 & 8.8 & 9.3 & 3.3 & 12.1 & 11.5 & 4.2 \\
\hline 2018 & 5.9 & 5.2 & 7.1 & 5.9 & 8.8 & 8.0 & 4.4 & 11.7 & 8.0 & 6.5 \\
\hline 2019 & 3.0 & 3.0 & 4.0 & 3.8 & 5.0 & 5.2 & 3.0 & 6.7 & 6.3 & 2.7 \\
\hline 2020 & 0.8 & 1.0 & 0.8 & 0.9 & 1.4 & 1.5 & 1.1 & 1.6 & 1.5 & 0.8 \\
\hline 2021 & 0.2 & 0.3 & 0.0 & 0.0 & 0.0 & 0.0 & 0.6 & 0.1 & 0.0 & 0.0 \\
\hline
\end{tabular}

\section{Most influential papers}

The most influential papers are determined based on the top ten papers with the highest citation number from the best ten fields in UTM (by publication count), i.e., Engineering (ENGI), Computer Science (COMP), Material Sciences (MATE), Physics and Astronomy (PHYS), Environmental Sciences (ENVI), Chemical Engineering (CENG), Mathematics (MATH), Energy (ENER), Chemistry (CHEM) and Social Sciences (SOCl). The most influential papers are listed in Figure S1 (Supplementary information). Apparently, some of the influential papers were ranked top five in more than one field, suggesting that the papers could be constituted as multidisciplinary. In addition, this finding also indicates the importance of multidisciplinary research to produce a more impactful publication. Review papers were excluded from this list regardless of the number of citations generated. Some of the review papers have to be removed manually due to the fact that Scopus did not categorize them as such.

The top ten most influential papers of UTM from top ten fields with the highest citation numbers is analysed in the present study. The most prominent keywords are extracted as Figure 4. There are two main research clusters that represent the most influential papers produced by UTM; (1) photovoltaicbased research which is a renewable energy cluster (the green cluster) and (2) materials science and engineering cluster (the red cluster) which are the constituent of engineering and materials science fields.

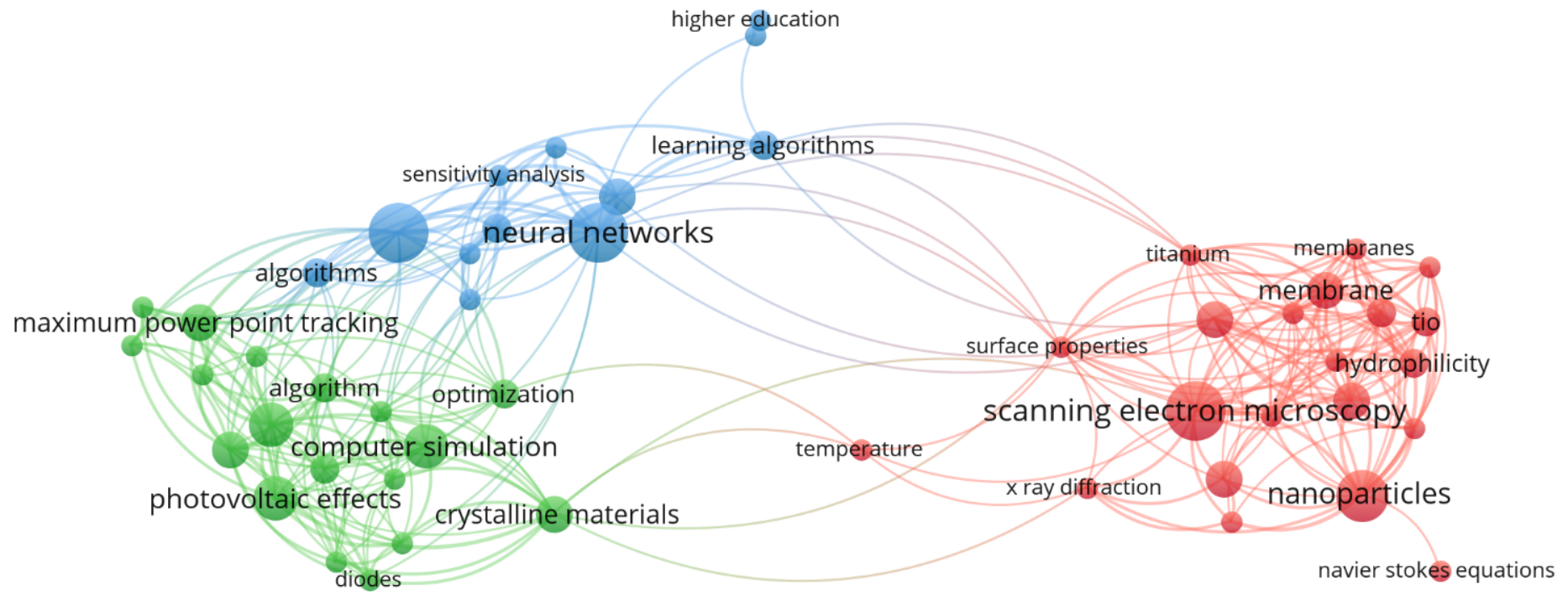

Figure 4. Co-occurrence of keywords from top ten of the most influential papers from top ten fields with the highest citation numbers. The shorter the distance between two nodes, the larger the number of co-occurrences of the two keywords. 


\section{Publication maturity}

Publications generally need time to generate citations, although the time required for the publication to reach its peak citation varies from publication to publication (evidently in our dataset, Table 8). National Taiwan University (NTU) and Peking University (PKU) reported the highest citations generated sooner than later, at three years and two years, respectively [12]. Taking only the top ten ASJC categories (sorted by the number of publications), further analysis was done to determine the "time of maturity" for publications published by UTM; looking specifically at the changes of the citation generated per paper (Table 8). In order to observe the change of average citation per paper, the "moving" average of the citation per paper was generated using the formula (1). The moving average concept here is a little different from the generalized moving average often seen in stock market analysis where the denominator (number of years) is fixed. On the contrary, the denominator used in the calculation here is dynamic, hence ENGI in 2013 would be:

$$
\begin{aligned}
& \underline{\sum_{n=\text { start year }}^{\text {end year }} \text { citation per } \text { paper }_{n}} \\
& n \\
& \frac{11.8+10.8+13.1+10.6}{4}=11.6
\end{aligned}
$$

All the moving averages for each ASJC category every year were calculated. A buffer of (+/-) $20 \%$ was set to observe how far off the citation per paper values (in Table 8) compared to the corresponding moving average in the same year. Publication in Computer sciences (COMP) exhibits significant deviation from the moving average in 2017 (Figure 5 at -34\%, more than the buffer set at -20\%). Since the cut-off of the study is limited to 2020, the "maturity" (defined as the minimum year needed by the publication to not deviate more than $20 \%$ from the moving average citation per paper) for UTM in Computer sciences (COMP) is five years as denoted in Figure 5.

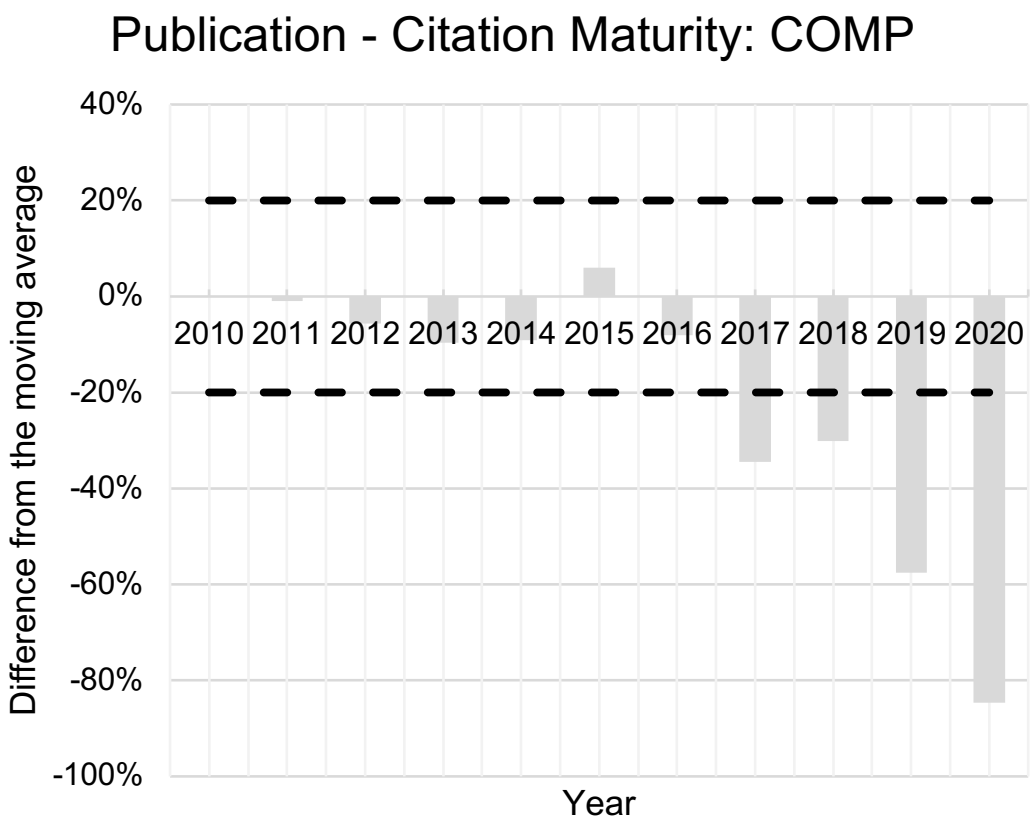

Figure 5. Publication - citation "maturity" analysis for COMP. 
Some ASJC categories exhibited anomalies, such as Social Science (SOCI, Figure 6) and Environmental Science (ENVI, Figure 7), where some fluctuation occurred. However, the maturity was determined by consistent "out-of-buffer-zone" behaviour shown by the chart, which resulted in the maturity of five years for SOCl and six years for ENVI. Most of the top ten ASJC categories need at least five to six years, as shown in Table 8, to generate substantial citations so that the citation per paper stays within the buffer zone, except for Chemical Science (CHEM). The maturity analysis could be helpful for the university to set a range years before "revisiting" the impact of the publication published, more importantly recognizing the difference of maturity by field of research.

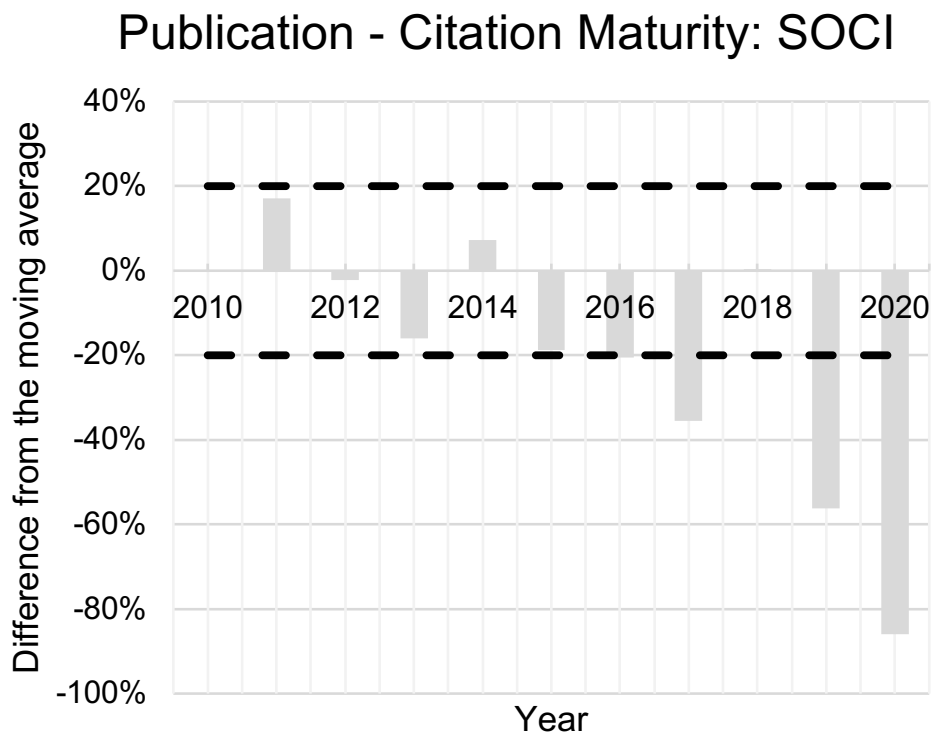

Figure 6. Publication - citation "maturity" analysis for SOCl.

\section{Publication - Citation Maturity: ENVI}

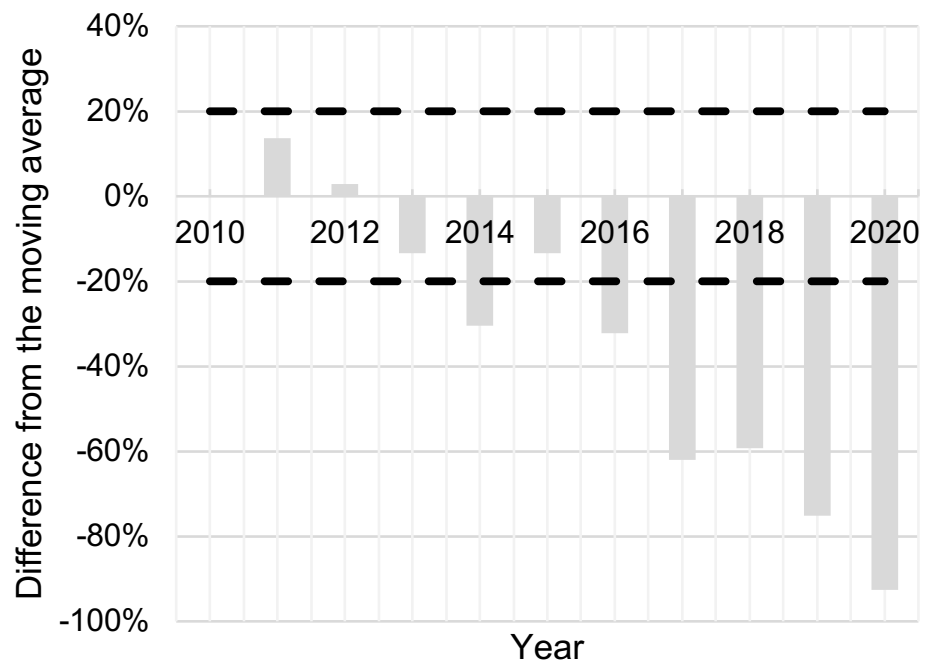

Figure 7. Publication - citation "maturity" analysis for ENVI. 
Table 8. Maturity for UTM top ten ASJC category.

\begin{tabular}{cc}
\hline ASJC Category & Maturity (Years) \\
\hline ENGI & 5 \\
COMP & 5 \\
MATE & 6 \\
PHYS & 6 \\
ENVI & 6 \\
CENG & 6 \\
MATH & 5 \\
ENER & 5 \\
CHEM & 7 \\
SOCI & 5 \\
\hline
\end{tabular}

\section{Cluster analysis}

Cluster analysis was executed on the entire dataset, on the 27 ASJC categories to determine the grouping based on two indicators; the number of citations and the number of publications. While the group in Table 4 is purely based on the preset ranges of publication volume, the clusters here are detected by the k-means algorithm, with a preset of five clusters. This results in five distinct groups (Figures 8, 9, and 10). Both Energy (ENER) and Chemistry (CHEM) were grouped in the first cluster, although ranked lower than Mathematics (MATH) in terms of publication volume due to their high value of citations generated. Mathematics (MATH) was grouped with the second cluster instead, while the rest of the fields were pretty much grouped similarly as in Table 4 . The cluster detected could be helpful for UTM to identify different strategies for different clusters in developing the related niche.

\section{First Cluster}

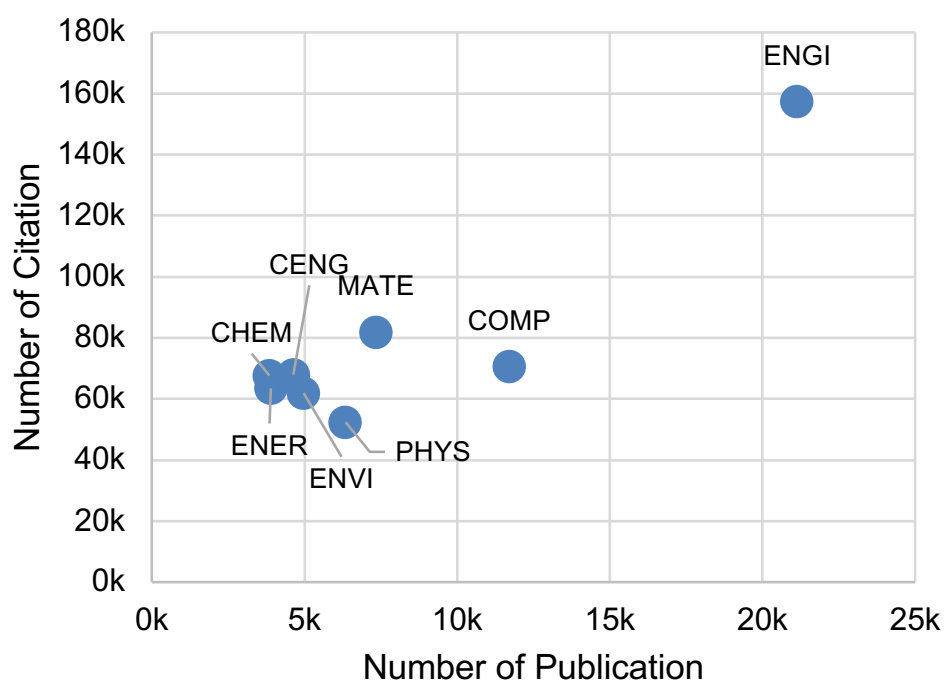

Figure 8. First cluster detected by k-means. 


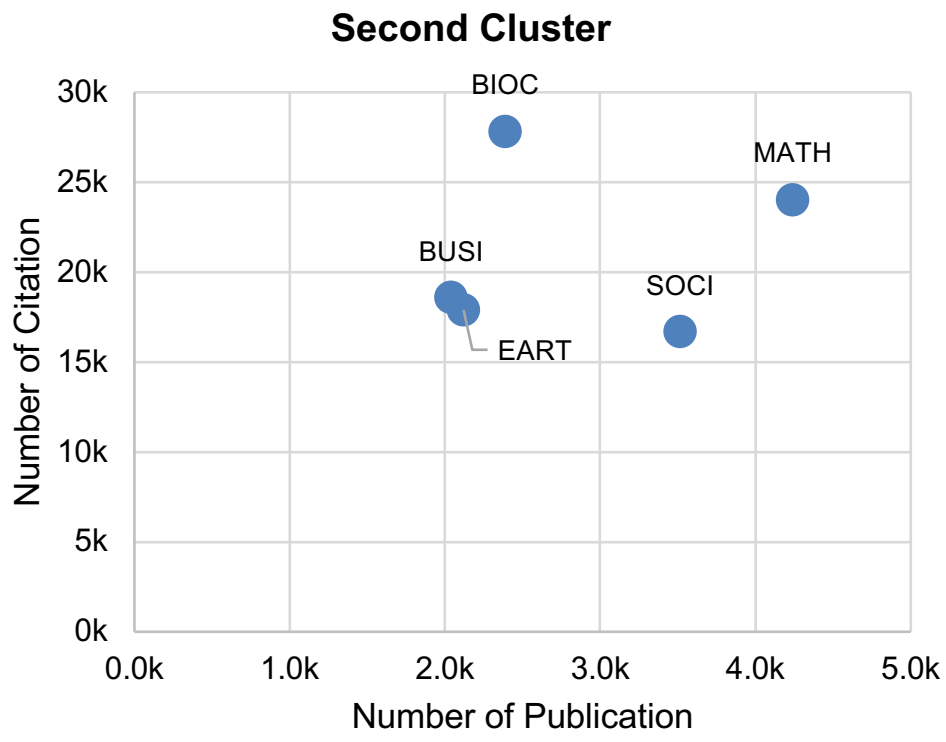

Figure 9. Second cluster detected by k-means.

\section{Third, Fourth and Fifth Clusters}

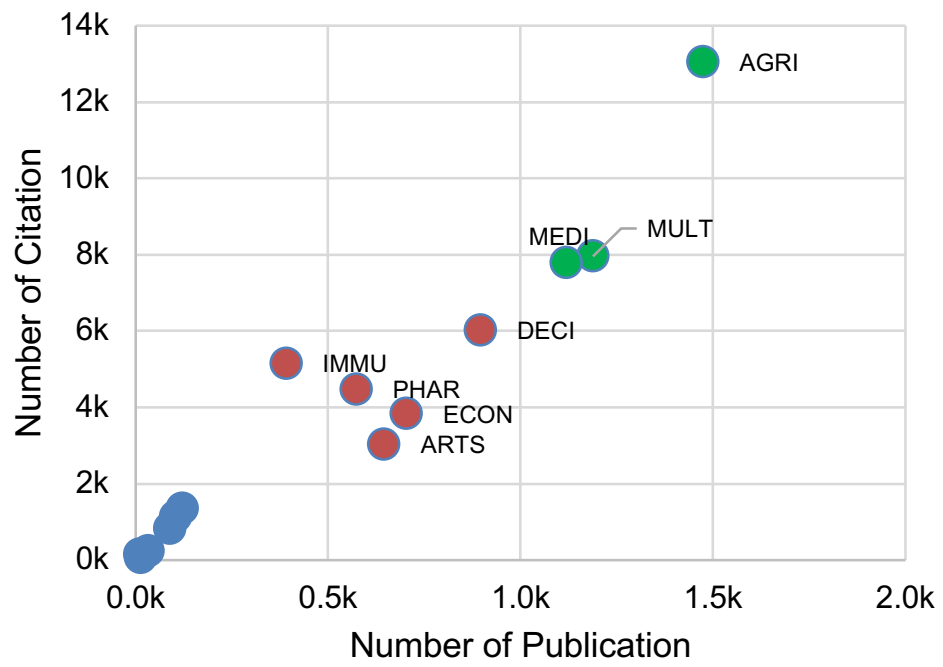

Figure 10. Third (green), fourth (orange) and fifth (blue) clusters detected by k-means

\section{Research factors analysis}

Further detailed analysis of the top ten fields in UTM is conducted in five research factors: number of publications, number of citations, number of authors, total equipment allocation, and research talents. The analysis of all the top ten fields in terms of all the five research factors is presented in Figure S3 (Supplementary info). Based on the data obtained as shown in Table 9, some important fields show a favourable citation impact despite having a relatively moderate publication. In addition, Mathematics (MATH) and Social Sciences (SOCl) are the two fields among the top ten fields in UTM that could be further improved in terms of publication outputs (i.e., number of publications and citations) despite having a reasonable number of authors and talents as indicated in Figure 11(a). Another significant finding here is that despite having a lower number of authors and publications, Materials Sciences (MATE) has a higher number of citations in comparison to those of Computer Science (COMP) which has $36 \%$ higher 
publication volume, as shown in Figure 11(b). In addition, Figure 11(b) also indicates that Material Sciences (MATE), Chemical Engineering (CHEM), Energy (ENER) and Chemistry (CHEM) have favourable citation numbers even though they possess relatively lower talents and publication numbers. This finding suggests the high potential of these four fields to obtain even more citations in the future, provided that the publication numbers of these fields can be further increased. Bear in mind that the number of citations could be increased by increasing the number of publications [13].

Table 9. The five research factors of the top ten ASJC categories in UTM. All values are normalized using the min-max approach.

\begin{tabular}{cccccc}
\hline ASJC Category & Authors & Publications & Citations & Talents & Equipment \\
\hline ENGI & 1.00 & 1.00 & 1.00 & 1.00 & 1.00 \\
COMP & 0.72 & 0.55 & 0.45 & 0.38 & 0.16 \\
MATE & 0.66 & 0.35 & 0.52 & 0.12 & 0.11 \\
PHYS & 0.63 & 0.30 & 0.33 & 0.04 & 0.04 \\
ENVI & 0.60 & 0.24 & 0.39 & 0.24 & 0.16 \\
CENG & 0.51 & 0.22 & 0.43 & 0.31 & 0.23 \\
MATH & 0.55 & 0.20 & 0.15 & 0.07 & 0.01 \\
ENER & 0.51 & 0.18 & 0.40 & 0.03 & 0.04 \\
CHEM & 0.44 & 0.18 & 0.43 & 0.07 & 0.07 \\
SOCI & 0.39 & 0.17 & 0.11 & 0.27 & 0.10 \\
\hline
\end{tabular}

(a)

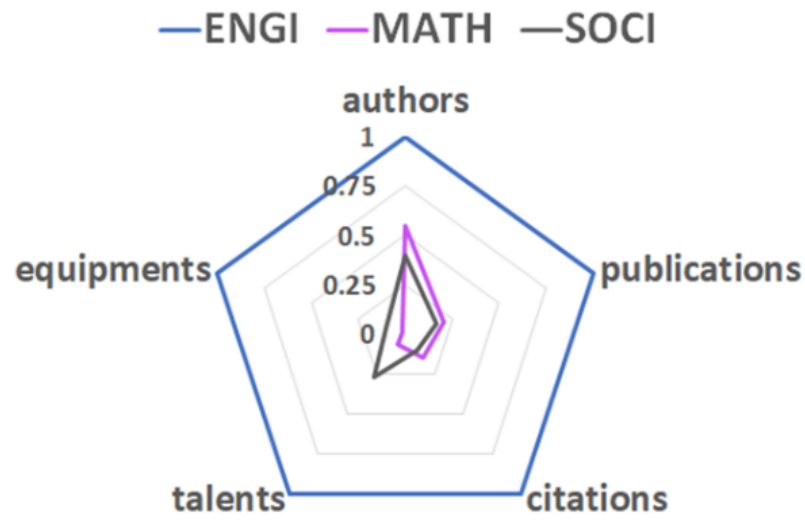

(b)

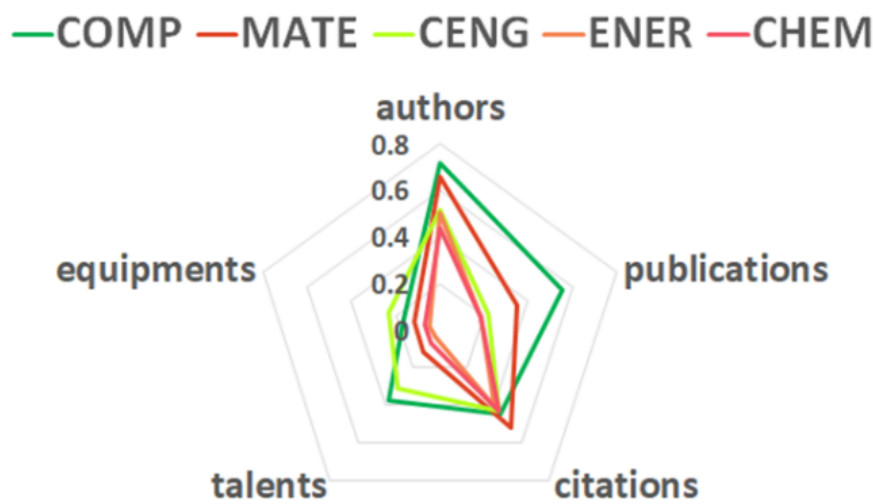

Figure 11. (a) The comparison between the engineering, mathematics and social science fields in terms of five research factors and (b) the analysis of research factors for materials science, chemical engineering, energy and chemistry fields.

\section{UTM's publications in comparison to other Malaysia RU}

This study also looks at the publication productivity of UTM in comparison to other Malaysia RU. Figure 12 depicts the total Scopus-indexed publications of all Malaysia RU beginning from the year 1996 to 2020. It can be deduced here that there is a remarkable increase in the publication trend starting the year 2007. This could be due to the research-university conferment to the RU in 2006, except for the UTM conferred its RU status in 2010. Despite all that, UTM publications have started to increase in 2007 and soared upwards more steeply after 2010 till 2015. Specifically, from the trend, it took about 3 years after 2010 (i.e., the conferment of RU status) for the UTM to be on a par with other RU. Then UTM needed another 3 years to surpass other RU except for Universiti Malaya (UM) in terms of publication as the UTM's publications thrived to the maximum from 2014 to 2015 . In 2019, UTM was the top university with the highest number of Scopus-index publications surpassing all the RU, including the UM. 


\section{Total Scopus-indexed publications of all RU in Malaysia from 1996 to 2020}

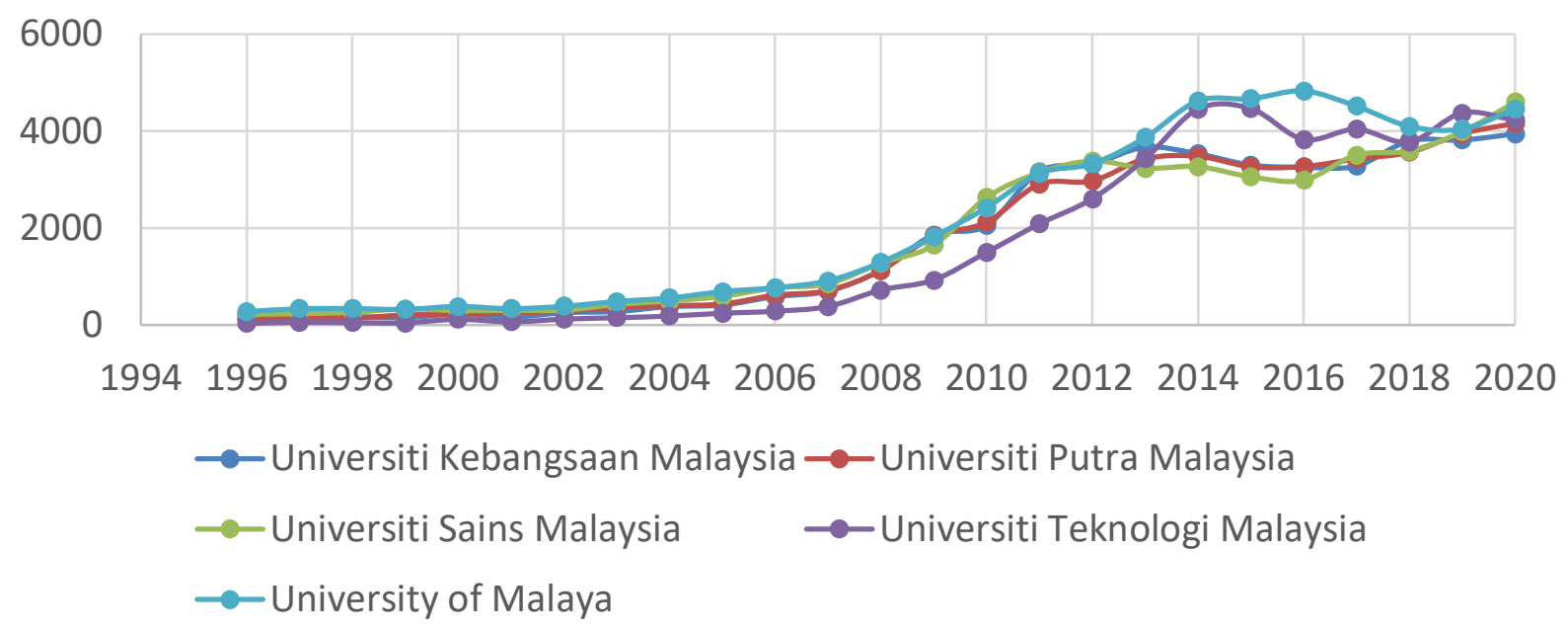

Figure 12. The total Scopus-indexed publications of UTM as compared to other RU in Malaysia from year 1996 to 2020.

In specific, there are four fields in which UTM propelled higher in a number of publications as compared to other RU from the year 2015 to 2020; 1) Computer science, 2) Engineering, 3) Energy, 4) Chemical engineering, as indicated in Figure S2 (Supplementary Info). As computer science and engineering fields are the top two in the number of publications among the fields in UTM, as previously shown in Table 2 , this result suggests that both fields are the prominent champion field of UTM over other RU thus far. Table 10 highlights the number of Scopus-indexed publications, highest subject area and top published journals of all Malaysian RU. UM is top of the group with 52,891 publications, followed by USM $(45,394)$ and UKM $(47,347)$. UTM ranks fifth in terms of total publication numbers with 42,170 publications. The highest publication area for all RU is engineering, except for UPM of which the agriculture field is the main subject area in publication. Most of the UM and USM's Scopus publication is in the Acta Crystallographica Section E Structure Reports Online journal, while UPM and UKM are in AIP Conference Proceedings journal. UTM has most of the publications in its journal, Jurnal Teknologi.

Table 10. Number of publications, highest subject area and top journals of all Malaysian RU from year 1996 to 2020.

\begin{tabular}{|c|c|c|c|c|}
\hline Research University & $\begin{array}{c}\text { No. of } \\
\text { publications } \\
(1996-2020)\end{array}$ & $\begin{array}{l}\text { Highest subject } \\
\text { area in } \\
\text { publications }\end{array}$ & $\begin{array}{l}\text { Second highest } \\
\text { subject area in } \\
\text { publications }\end{array}$ & Top Journals \\
\hline Universiti Malaya & 52,891 & Engineering (ENG) & Medicine (MEDI) & $\begin{array}{c}\text { Acta Crystallographica Section } \\
\text { E Structure Reports Online } \\
(2,259)\end{array}$ \\
\hline $\begin{array}{l}\text { Universiti Teknologi } \\
\text { Malaysia }\end{array}$ & 42,170 & Engineering (ENG) & $\begin{array}{l}\text { Computer science } \\
\text { (COMP) }\end{array}$ & Jurnal Teknologi $(1,626)$ \\
\hline Universiti Putra Malaysia & 43,391 & Agriculture (AGRI) & Engineering (ENG) & $\begin{array}{l}\text { AIP Conference Proceedings } \\
\qquad(595)\end{array}$ \\
\hline $\begin{array}{c}\text { Universiti Kebangsaan } \\
\text { Malaysia }\end{array}$ & 43,747 & Engineering (ENG) & Medicine (MEDI) & $\begin{array}{l}\text { AIP Conference Proceedings } \\
\qquad(1,613)\end{array}$ \\
\hline Universiti Sains Malaysia & 45,394 & Engineering (ENG) & $\begin{array}{l}\text { Materials science } \\
\text { (MATE) }\end{array}$ & $\begin{array}{c}\text { Acta Crystallographica Section } \\
\text { E Structure Reports Online } \\
(1,796)\end{array}$ \\
\hline
\end{tabular}




\section{Conclusion}

Based on the above discussion, UTM has great strength in several important fields such as engineering, computer sciences, materials science, chemical engineering, energy, environmental science and physics and astronomy in terms of publication outputs (i.e., number of publications and citations). Among the top 10 fields in UTM, materials science, chemical engineering, energy and chemistry areas are seen to have a high potential to further excel in citation provided that the publication numbers of these fields are further increased. The publications outputs (i.e., number of publications and citation) for some UTM fields are still considered low despite showing a promising publication growth in recent years and having a reasonable number of authors and talents. Besides, the top published journals of all the research universities (RU) could describe the impact of the research. On that account, the UTM research can be further enhanced in terms of quality to be published in a more impactful and prestigious journal. There is a need to enhance research quality to obtain a higher publication impact. Based on all the universities' top published journals, there is still plenty of room for research improvement to enhance the RU's publication impact, including UTM.

\section{Acknowledgments}

We are grateful to Siti Nur Sakinah Ahmad, Wong Yah Jin, Nurfahain Mohamed Rusli, Nadia Hartini Mohd Adzmi, and Rozita Abdul Jalil for their help with data organization. The views and conclusions contained herein are those of the authors and should not be interpreted as necessarily representing the official policies or endorsements, either expressed or implied, of Universiti Teknologi Malaysia.

\section{References}

[1] Karno M, Shamsir S, Buntat Y, and Sharuddin N, A bibliometric analysis of the research output of Malaysia research universities scholarly publication indexed in Scopus from $2006-2015,6^{\text {th }}$ International Conferences on Libraries, Information and Society (ICOLIS 2016). 2016.

[2] Suryani I, Yaacob A, Abd Aziz NH, Abd Rashid S, and Desa H. Research publication output by academicians in public and private universities in Malaysia. The Journal of Higher Education, 2013, 2, 84-90.

[3] van Leeuwen TN, van Wijk E, and Wouters PF. Bibliometric analysis of output and impact based on CRIS data: a case study on the registered output of a Dutch university. Scientometrics, 2016, 106, 1-16.

[4] Boukacem-Zeghmouri C, Bador P, Lafouge $\mathrm{T}$, and Prost $\mathrm{H}$. Relationships between consumption, publication and impact in French universities in a value perspective: A bibliometric analysis. 2016, 106, (1), 263-280.

[5] Cancino CA, Merigó JM, and Coronado FC. A bibliometric analysis of leading universities in innovation research. Journal of Innovation \& Knowledge, 2017, 2, (3), 106-124.

[6] Khatun R, Xiang $\mathrm{H}$, Yang $\mathrm{Y}$, Wang J, and Yildiz G. Bibliometric analysis of research trends on the thermochemical conversion of plastics during 1990-2020. Journal of Cleaner Production, 2021, 317, 128373.

[7] Shi J, Gao Y, Ming L, Yang K, Sun Y, Chen J, Shi S, Geng J, Li L, Wu J, and Tian J. A bibliometric analysis of global research output on network meta-analysis. BMC Medical Informatics and Decision Making, 2021, 21, (1), 144.

[8] Meo SA, Usmani AM. Impact of R\&D expenditures on research publications, patents and high-tech exports among European countries. European Review for Medical and Pharmacological Sciences, 2014, 18 1, 1-9.

[9] Zeb A, Liu W, Wu J, Lian J, and Lian Y. Knowledge domain and emerging trends in nanoparticles and plants interaction research: A scientometric analysis. Nanolmpact, 2021, 21, 100278.

[10] Zhang JZ, Srivastava PR, Sharma D, and Eachempati P. Big data analytics and machine learning: A retrospective overview and bibliometric analysis. Expert Systems with Applications, 2021, 184, 115561.

[11] Malaysian Science and Technology Information Centre (MASTIC). Malaysian Research and Development Classification System (6 ${ }^{\text {th }}$ Edition), $6^{\text {th }}$ ed. Ministry of Science, Technology and Innovation (MOSTI), 2011.

[12] Ming HW, Hui ZF, and Yuh SH. Comparison of universities' scientific performance using bibliometric indicators. Malaysian Journal of Library \& Information Science, 2011, 16, (2), 1-19.

[13] Simko I. Analysis of bibliometric indicators to determine citation bias. Palgrave Communications, 2015, 1, (1), 15011. 

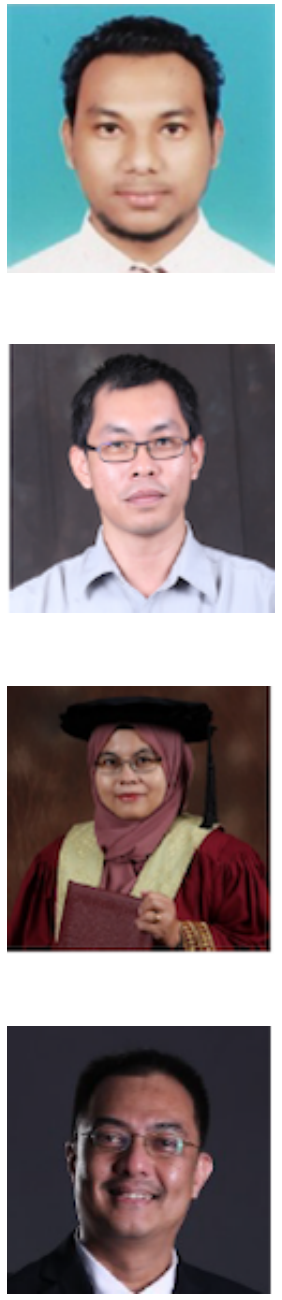

Abdul Hakim Md Yusop graduated with Ph.D. in Biomedical Engineering at Universiti Teknologi Malaysia. He has been involved in materials science and engineering fields since 2011 and his recent research focuses on the development of bone scaffolds made of biodegradable metals, particularly ironbased materials. Currently, he serves as a post-doctoral fellow at Ibnu Sina Institute for Scientific and Industrial Research, Universiti Teknologi Malaysia.

Teong Han Chew is a research officer with over 10-year experience in research project management and data analytics with the current interest in task automation. Competent in Python programming and KNIME analytics platform. Currently overseeing the research-related performance of the entire university and assisting in research \& innovation strategies management and performance tracking.

Fadzlin Md Sairan is currently a Senior Research Officer at Ibnu Sina Institute for Scientific and Industrial Research, Universiti Teknologi Malaysia (UTM), Johor Bahru. Previously she worked as a research officer at the Institute of Environmental and Water Resource Management, UTM Johor Bahru (20012008) and has conducted several research and consultancy projects, funded by various agencies, including a grant from European Commission on water sustainability.

Hadi Nur is a Professor and Institute Director of Ibnu Sina Institute for Scientific and Industrial Research (ISI-SIR), Universiti Teknologi Malaysia (UTM), and a Professor at Universitas Negeri Malang (UM), Indonesia. 